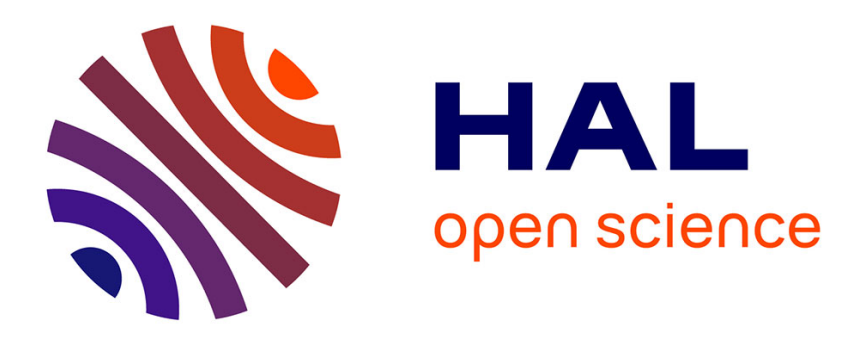

\title{
Numerical approximation of some time optimal control problems
}

Marius Tucsnak, Julie Valein, Chi-Ting Wu

\section{To cite this version:}

Marius Tucsnak, Julie Valein, Chi-Ting Wu. Numerical approximation of some time optimal control problems. European Control Conference, Jul 2015, Linz, Austria. pp.ThA3.4, 10.1109/ECC.2015.7330724 . hal-01246356

\section{HAL Id: hal-01246356 \\ https://hal.science/hal-01246356}

Submitted on 18 Dec 2015

HAL is a multi-disciplinary open access archive for the deposit and dissemination of scientific research documents, whether they are published or not. The documents may come from teaching and research institutions in France or abroad, or from public or private research centers.
L'archive ouverte pluridisciplinaire HAL, est destinée au dépôt et à la diffusion de documents scientifiques de niveau recherche, publiés ou non, émanant des établissements d'enseignement et de recherche français ou étrangers, des laboratoires publics ou privés. 


\title{
Numerical approximation of some time optimal control problems
}

\author{
Marius Tucsnak ${ }^{1}$, Julie Valein ${ }^{1}$ and Chi-Ting $\mathrm{Wu}^{1}$ \\ *
}

\begin{abstract}
In this work we study the numerical approximation of the solutions of a class of abstract parabolic time optimal control problems. Our main results assert that, provided that the target is a closed ball centered at the origin and of positive radius, the optimal time and the optimal controls of the approximate time optimal problems converge to the optimal time and to the optimal controls of the original problem. In order to prove our main theorem, we provide a nonsmooth data error estimate for abstract parabolic systems.
\end{abstract}

Keywords. distributed parameter systems, optimal control, numerical approximation

\section{Introduction}

Time optimal control of infinite dimensional systems is a subject of growing interest, motivated by numerous applications in domains such as guidance of complex systems or temperature regulation in large buildings. In recent year, using new tools from infinite dimensional systems theory, the literature devoted to this topic grew in a considerable manner (see [1]-[11] and references therein). The specific case of time optimal control for systems governed by parabolic PDE's has numerous applications, from which we quote optimization of building thermal storage (see, for instance [7] and references therein).

The aim of this paper is to study the approximation of the solutions of time optimal control problems for a class of infinite dimensional linear systems by projecting the original problem on an appropriate family of finite dimensional spaces. This is a delicate question since, as shown in the above mentioned references, time

\footnotetext{
${ }^{*}$ Institut Élie Cartan de Lorraine, INRIA, Nancy-Université de Lorraine, CNRS, B.P. 70239, F-54506 Vandoeuvre-lsNancy Cedex, France (marius.tucsnakeuniv-lorraine.fr, julie.valein@univ-lorraine.fr, chi-ting.wu@univ-lorraine.fr)
}

optimal controls are usually highly oscillating functions (due to the bang-bang property). As far as we know, the only papers having already investigated this issue are [15] and [16], which investigated finite elements approximation for systems governed by the heat equation.

To be more precise, let $X$ and $U$ be real Hilbert spaces, and let $A_{0}: \mathscr{D}\left(A_{0}\right) \rightarrow X$ be a strictly positive operator with compact resolvents. It is known that $-A_{0}$ generates an exponentially stable analytic semigroup, denoted by $\mathbb{T}$. For $\gamma>0$ we denote by $X_{\gamma}$ the space $\mathscr{D}\left(A_{0}^{\gamma}\right)$, endowed with the graph norm. For $\gamma<0, X_{\gamma}$ stands for the dual of $X_{-\gamma}$ with respect to the pivot space $X$. We also introduce an operator $B \in \mathscr{L}\left(U, X_{-\alpha}\right)$ with $0 \leqslant \alpha \leqslant \frac{1}{2}$, called control operator. In this paper we consider time optimal control problems for the following system,

$$
\begin{aligned}
\dot{z}(t)+A_{0} z(t) & =B u(t) & (t \geq 0), \\
z(0) & =z_{0} & \left(z_{0} \in X\right),
\end{aligned}
$$

where $u \in L^{\infty}([0, \infty[; U)$. Using the notation in [12], the solution of (1)-(2) writes :

$$
z(t)=\mathbb{T}_{t} z_{0}+\Phi_{t} u
$$

where

$$
\Phi_{t} u=\int_{0}^{t} \mathbb{T}_{t-\sigma} B u(\sigma) d \sigma .
$$

Given $\varepsilon>0$, denote by $\bar{B}(0, \varepsilon)$ the closed ball centered in zero and of radius $\varepsilon$ in $X$. We consider the time optimal control problem which consists in determining the smallest $\tau_{0}^{*}>0$ such that there exists $u$ with $\|u\|_{L^{\infty}([0, \tau] ; U)} \leqslant 1$ and where the solution $z$ of (1)-(2) satisfies $z(\tau) \in \bar{B}(0, \varepsilon)$. The corresponding optimal control is denoted by $u_{0}^{*}$.

Denote

$$
\mathscr{U}_{\mathrm{ad}}=\left\{u \in L ^ { \infty } \left(\left[0, \infty[; U) \mid\|u\|_{L^{\infty}([0, \infty[; U)} \leqslant 1\right\} .\right.\right.
$$

We call $u \in \mathscr{U}_{\text {ad }}$ an admissible control if there exists $\tau>0$ such that $\mathbb{T}_{\tau} z_{0}+\Phi_{\tau} u \in \bar{B}(0, \varepsilon)$. It is well-known that the above optimal time $\tau_{0}^{*}$ and optimal control $u_{0}^{*}$ 
exist and that, under additional assumptions, they are unique.

Let $\left(V_{h}\right)_{h>0}$ be a family of finite dimensional subspaces of $X_{\frac{1}{2}}$ and let $U_{h}=B^{*} V_{h}$. These spaces are normed spaces endowed with the restriction of the norm of $X_{\frac{1}{2}}$ (resp. $U$ ). We denote $P_{h}$ (resp. $Q_{h}$ ) the orthogonal projector from $X$ onto $V_{h}$ (resp. $U$ onto $U_{h}$ ). For each $h>0$, we consider the following system:

$$
\begin{aligned}
\dot{z}_{h}(t)+A_{h} z_{h}(t) & =B_{h} u_{h}(t) \quad(t \geq 0), \\
z_{h}(0) & =P_{h} z_{0},
\end{aligned}
$$

where $\left(A_{h}\right)_{h>0}$ is defined by

$$
\left\langle A_{h} \varphi, \psi\right\rangle=\left\langle A_{0}^{\frac{1}{2}} \varphi, A_{0}^{\frac{1}{2}} \psi\right\rangle
$$

for every $\varphi, \psi \in V_{h}$. Moreover, $B_{h} \in \mathscr{L}\left(U, V_{h}\right)$ is defined by:

$$
\left\langle B_{h} u, \varphi\right\rangle=\left\langle u, B^{*} \varphi\right\rangle_{U},
$$

for every $\varphi \in V_{h}, u \in U$. The above system is the Galerkin approximation of (1)-(2).

Denote by $\bar{B}_{h}(0, \varepsilon)$ the closed ball centered in zero in $V_{h}$ with radius $\varepsilon$. For each $h>0$, we consider the time optimal control problem for the above system (3)-(4) which is to determine the smallest $\tau_{h}^{*}>0$ such that there exists $u_{h}$ with $\left\|u_{h}\right\|_{L^{\infty}\left([0, \tau] ; U_{h}\right)} \leqslant 1$ and $z_{h}(\tau) \in \bar{B}_{h}(0, \varepsilon)$. Moreover, we aim to determine the corresponding optimal controls $u_{h}^{*}$.

The goal of this work is to study the convergence of $\tau_{h}^{*}$ to $\tau_{0}^{*}$ and of $u_{h}^{*}$ to $u_{0}^{*}$ when $h \rightarrow 0$. To this aim, we need appropriate assumptions on the approximation properties of the spaces $\left(V_{h}\right)_{h>0}$ and $\left(U_{h}\right)_{h>0}$. More precisely, we assume that there exist $\theta>0, h_{1}>$ $0, C>0,0 \leqslant \beta \leqslant \alpha$, such that for every $h \in\left(0, h_{1}\right)$ and $0 \leqslant \gamma \leqslant 1$, we have:

$$
\begin{aligned}
& \left\|x-P_{h} x\right\|_{X} \leqslant C h^{\theta \gamma}\|x\|_{\gamma} \text { for every } x \in X_{\gamma} . \\
& \left\|\left(I-P_{h}\right) B\right\|_{\mathscr{L}(U, X)} \leqslant C h^{\theta(1-\beta)} . \\
& \left\|P_{h} B\right\|_{\mathscr{L}\left(U, V_{h}\right)} \leqslant C h^{-\theta \beta} . \\
& \lim _{h \rightarrow 0}\left\|Q_{h} u-u\right\|_{U}=0 \text { for every } u \in U .
\end{aligned}
$$

Note that assumptions $(C 1)-(C 4)$ are very natural when applying our results to systems governed by parabolic partial differential equations. They are satisfied, in particular, by all the usual approximations schemes based on finite elements or finite differences. To be more precise, with assumptions $(C 1)-(C 4)$, we can prove the following approximation results with rough initial data: $\forall z_{0} \in X, \exists \hat{h}>0, C>0$, s.t. $\forall h \in$ $(0, \hat{h}), \forall t \geqslant 0$,

$$
\begin{aligned}
\| z\left(t, z_{0}, u\right)- & z_{h}\left(t, P_{h} z_{0}, u\right)\left\|\leqslant C h^{\theta} t^{-1}\right\| z_{0} \|_{X} \\
& +C h^{\theta(1-\beta)}|\ln h|\|u\|_{L^{\infty}([0, T] ; U)} .
\end{aligned}
$$

We refer to [9] and [2] for more details about the above approximation.

We are now in position to state the main results of this paper:

Theorem 1. With the above notation and assumptions, assume that $z_{0} \in X,\left\|z_{0}\right\|>\varepsilon$ and that $(C 1)-(C 4)$ hold. Then $\lim _{h \rightarrow 0} \tau_{h}^{*}=\tau_{0}^{*}$.

Theorem 2. With the above notation and assumptions, assume that if $z \in X$ is such that the measure of the set of those $t \geqslant 0$ such that $B^{*} \mathbb{T}_{t} z=0$ is strictly positive then $z=0$. Then we have

$$
u_{h}^{*} \rightarrow u_{0}^{*} \text { strongly in } L^{2}\left(\left[0, \tau^{*}\right] ; U\right) .
$$

\section{Sketch of the proof.}

\subsection{Proof of Theorem 1}

The proof of our main results requires several steps, which will be briefly described in this section.

Lemma 1. With the above notation and assumptions, let $\lambda_{1}$ (resp. $\lambda_{1, h}$ ) be the smallest eigenvalue of $A_{0}$ (resp. of $A_{h}$ ). Denote $z_{0, h}=P_{h} z_{0}$. Then

(i) $\tau_{0}^{*}\left(z_{0}\right) \leqslant \frac{\ln \left(\left\|z_{0}\right\| / \varepsilon\right)}{\lambda_{1}}, \quad\left(z_{0} \in X\right)$.

(ii) $\tau_{h}^{*}\left(z_{0, h}\right) \leqslant \frac{\ln \left(\left\|z_{0, h}\right\| / \varepsilon\right)}{\lambda_{1, h}}, \quad\left(z_{0, h} \in V_{h}\right)$.

(iii) $\lambda_{1} \leqslant \lambda_{1, h}$

Proof. We prove at first $(i)$. It suffices to notice that:

$$
\left\|z\left(t, z_{0}, 0\right)\right\| \leqslant e^{-\lambda_{1} t}\left\|z_{0}\right\| .
$$

Then, by taking $t=\frac{\ln \left(\left\|z_{0}\right\| / \varepsilon\right)}{\lambda_{1}}$, we have: $\left\|z\left(t, z_{0}, 0\right)\right\| \leqslant \varepsilon$. This proves $(i)$.

A similar argument shows that (ii) also holds.

We end by proving (iii). In fact, this inequality is easily deduced by the min-max formula:

$$
\lambda_{1}=\min _{z \in X_{\frac{1}{2}}} \frac{\left\|A_{0}^{\frac{1}{2}} z\right\|^{2}}{\|z\|^{2}}
$$

and

$$
\lambda_{1, h}=\min _{z \in V_{h}} \frac{\left\|A_{0}^{\frac{1}{2}} z\right\|^{2}}{\|z\|^{2}} .
$$


We also need the following result

Lemma 2. With the notation and assumptions in Lemma 1 , for every $z_{0} \in X,\left\|z_{0}\right\|>\varepsilon$, there exist $c, C>0, \tilde{h}>0$ such that for any $h \in(0, \tilde{h})$, we have

$$
c \leqslant \tau_{h}^{*}\left(P_{h} z_{0}\right) \leqslant C,
$$

where $C=\frac{2 \ln \left(\left\|z_{0}\right\| / \varepsilon\right)}{\lambda_{1}}$.

Proof. We begin by proving that $\tau_{h}^{*}\left(P_{h} z_{0}\right)$ is bounded from below. Suppose by contradiction that $\lim _{h \rightarrow 0} \tau_{h}^{*}\left(P_{h} z_{0}\right)=0$. By the continuity of $t \mapsto z_{h}(t)$, we have:

$$
\begin{gathered}
\lim _{h \rightarrow 0}\left\|z_{h}\left(\tau_{h}^{*}, P_{h} z_{0}, u_{h}^{*}\right)-z_{h}\left(0, P_{h} z_{0}, u_{h}^{*}\right)\right\| \\
=\lim _{h \rightarrow 0}\left\|z_{h}\left(\tau_{h}^{*}, P_{h} z_{0}, u_{h}^{*}\right)-P_{h} z_{0}\right\|=0 .
\end{gathered}
$$

Using the fact that $\left\|z_{h}\left(\tau_{h}^{*}, P_{h} z_{0}, u_{h}^{*}\right)\right\| \leqslant \varepsilon$, it is clear that $\lim _{h \rightarrow 0}\left\|P_{h} z_{0}\right\| \leqslant \varepsilon$. However, with $(C 1)$ it is clear that:

$$
\lim _{h \rightarrow 0}\left\|P_{h} z_{0}-z_{0}\right\|=0,
$$

which leads to the contradiction with the fact that $\left\|z_{0}\right\|>\varepsilon$.

We prove now that $\tau_{h}^{*}\left(P_{h} z_{0}\right)$ is bounded from above. This is obvious by using Lemma 1 , since

$$
\tau_{h}^{*}\left(P_{h} z_{0}\right) \leqslant \frac{\ln \left(\left\|P_{h} z_{0}\right\| / \varepsilon\right)}{\lambda_{1, h}} \leqslant \frac{2 \ln \left(\left\|z_{0}\right\| / \varepsilon\right)}{\lambda_{1}}<+\infty .
$$

Proof of Theorem 1. It suffices to prove the following two inequalities:

$$
\begin{gathered}
\liminf _{h \rightarrow 0} \tau_{h}^{*} \geqslant \tau_{0}^{*}, \\
\limsup _{h \rightarrow 0} \tau_{h}^{*} \leqslant \tau_{0}^{*} .
\end{gathered}
$$

We begin by proving (8). We first notice that, for every $T>0$ and $u \in \mathscr{U}_{a d}$ :

$$
\tau_{0}^{*}\left(z_{0}\right) \leqslant T+\tau_{0}^{*}\left(z\left(T, z_{0}, u\right)\right)
$$

By (7), we have

$$
\begin{gathered}
\left\|z\left(\tau_{h}^{*}, z_{0}, u_{h}^{*}\right)-z_{h}\left(\tau_{h}^{*}, P_{h} z_{0}, u_{h}^{*}\right)\right\| \\
\leqslant C h^{\theta} \tau_{h}^{*-1}\left\|z_{0}\right\|_{X}+C h^{\theta(1-\beta)}|\ln h|\|u\|_{L^{\infty}([0, T] ; U)}
\end{gathered}
$$

This leads to:

$$
\begin{gathered}
\left\|z\left(\tau_{h}^{*}, z_{0}, u_{h}^{*}\right)\right\| \leqslant \varepsilon+C h^{\theta} \tau_{h}^{*-1}\left\|z_{0}\right\|_{X} \\
+C h^{\theta(1-\beta)}|\ln h|\|u\|_{L^{\infty}([0, T] ; U)} \\
\leqslant \varepsilon+C h^{\theta} \tau_{h}^{*-1}+C h^{\theta(1-\beta)}|\ln h| .
\end{gathered}
$$

Denote $\overline{z_{0}}=z\left(\tau_{h}^{*}, z_{0}, u_{h}^{*}\right)$. According to (10) with $T=$ $\tau_{h}^{*}$, we have:

$$
\tau_{0}^{*}\left(z_{0}\right) \leqslant \tau_{h}^{*}+\tau_{0}^{*}\left(\overline{z_{0}}\right) .
$$

In fact, $u_{h}^{*} \in L^{\infty}\left(\left[0,+\infty\left[; U_{h}\right) \subset L^{\infty}([0,+\infty[; U)\right.\right.$ and $\left\|u_{h}^{*}(t)\right\| \leqslant 1$ which means that $u_{h}^{*}$ is an admissible control for the original system.

Then, according to Lemma 1, we have:

$$
\begin{aligned}
& \tau_{0}^{*} \leqslant \tau_{h}^{*}+\frac{\ln \left(\left(\varepsilon+C h^{\theta} \tau_{h}^{*-1}+C h^{\theta(1-\beta)}|\ln h|\right) / \varepsilon\right)}{\lambda_{1}} \\
& \leqslant \tau_{h}^{*}+\frac{C h^{\theta}}{\lambda_{1} \varepsilon} \tau_{h}^{*-1}+C \frac{h^{\theta(1-\beta)}|\ln h|}{\lambda_{1} \varepsilon} .
\end{aligned}
$$

Thus, (8) can be deduced by taking $h$ to zero and by the fact that $\lim _{h \rightarrow 0} \tau_{h}^{*}>c>0$ (Lemma 2).

We now prove the second inequality (9). We have:

$$
\begin{gathered}
\left\|z_{h}\left(\tau_{0}^{*}, P_{h} z_{0}, Q_{h} u^{*}\right)-z\left(\tau_{0}^{*}, z_{0}, u^{*}\right)\right\| \\
\leqslant\left\|z_{h}\left(\tau_{0}^{*}, P_{h} z_{0}, Q_{h} u^{*}\right)-z_{h}\left(\tau_{0}^{*}, P_{h} z_{0}, u^{*}\right)\right\| \\
+\left\|z_{h}\left(\tau_{0}^{*}, P_{h} z_{0}, u^{*}\right)-z\left(\tau_{0}^{*}, z_{0}, u^{*}\right)\right\| \\
\leqslant\left\|z_{h}\left(\tau_{0}^{*}, P_{h} z_{0}, Q_{h} u^{*}\right)-z_{h}\left(\tau_{0}^{*}, P_{h} z_{0}, u^{*}\right)\right\| \\
+C h^{\theta} \tau_{0}^{*-1}+C h^{\theta(1-\beta)}|\ln h| .
\end{gathered}
$$

Set $f(h)=\left\|z_{h}\left(\tau_{0}^{*}, P_{h} z_{0}, Q_{h} u^{*}\right)-z_{h}\left(\tau_{0}^{*}, P_{h} z_{0}, u^{*}\right)\right\|$. We notice that $\lim _{h \rightarrow 0} f(h)=0$. Indeed,

$$
\begin{gathered}
\lim _{h \rightarrow 0}\left\|z_{h}\left(\tau_{0}^{*}, P_{h} z_{0}, Q_{h} u^{*}\right)-z_{h}\left(\tau_{0}^{*}, P_{h} z_{0}, u^{*}\right)\right\| \\
=\lim _{h \rightarrow 0}\left\|\Phi_{\tau_{0}^{*}, h}\left(u^{*}-Q_{h} u^{*}\right)\right\| .
\end{gathered}
$$

Since $\Phi_{\tau_{0}^{*}, h} \in \mathscr{L}\left(L^{2}\left(0, \tau_{0}^{*} ; U\right), X\right)$ (by the admissibility assumption upon $B$ ), this leads to:

$$
\lim _{h \rightarrow 0} f(h) \leqslant K \lim _{h \rightarrow 0}\left\|Q_{h} u^{*}-u^{*}\right\|_{L^{2}\left(0, \tau_{0}^{*} ; U\right)} \rightarrow 0,
$$

using (C4). Thus, we have:

$$
\begin{gathered}
\left\|z_{h}\left(\tau_{0}^{*}, P_{h} z_{0}, Q_{h} u_{h}^{*}\right)\right\| \leqslant \varepsilon+f(h) \\
+C h^{\theta} \tau_{0}^{*-1}+C h^{\theta(1-\beta)}|\ln h| .
\end{gathered}
$$

By the similar argument as in (11), we have:

$$
\begin{gathered}
\tau_{h}^{*} \leqslant \tau_{0}^{*} \\
+\frac{\ln \left(\left(\varepsilon+f(h)+C h^{\theta} \tau_{0}^{*-1}+C h^{\theta(1-\beta)}|\ln h|\right) / \varepsilon\right)}{\lambda_{1, h}} \\
\leqslant \tau_{0}^{*}+\frac{f(h)+C h^{\theta} \tau_{0}^{*-1}+C h^{\theta(1-\beta)}|\ln h|}{\lambda_{1} \varepsilon} .
\end{gathered}
$$

This leads to inequality (9) by letting $h$ tend to zero. 


\subsection{Proof of Theorem 2}

Before giving the proof, we recall a standard energy estimate.

Lemma 3. Assume that $z_{0} \in X_{\frac{1}{2}-\alpha}$. Then, there exists $c>0$ such that

$$
\begin{gathered}
\|z(\tau)\|_{\frac{1}{2}-\alpha}^{2}+\int_{0}^{\tau}\left(\|\dot{z}(s)\|_{-\alpha}^{2}+\|z(s)\|_{1-\alpha}^{2}\right) \mathrm{d} s \\
\leqslant C\left(\int_{0}^{\tau}\|B u(s)\|_{-\alpha}^{2} d s+\left\|z_{0}\right\|_{\frac{1}{2}-\alpha}^{2}\right) .
\end{gathered}
$$

\section{Proof of Theorem 2.}

Denote $T=2 \frac{\ln \left(\left\|z_{0}\right\| / \varepsilon\right)}{\lambda_{1}}$. It is clear that $\tau_{h}^{*} \leqslant T$ for all $h>0$ and $\tau_{0}^{*} \leqslant T$. We extend $\left(u_{h}^{*}\right)_{h}$ and $\left(u_{0}^{*}\right)$ to time $T$ by zero.

Since $\left\|u_{h}^{*}\right\|_{L^{\infty}(0, T ; U)} \leqslant 1$, there exist a control $\bar{u} \in$ $L^{\infty}(0, T ; U)$ and a subsequence $\left(h_{n}\right)_{n} \rightarrow 0$, such that :

$$
u_{h_{n}}^{*} \rightarrow \bar{u} \quad \text { weakly } * \quad \text { in } \quad L^{\infty}(0, T ; U)
$$

Now we prove that $\bar{u}=u_{0}^{*}$.

The main step here is to prove the following convergence property :

$$
\left\|z_{h_{n}}\left(\tau_{h_{n}}^{*}, u_{h_{n}}^{*}, P_{h} z_{0}\right)-z\left(\tau_{0}^{*}, \bar{u}, z_{0}\right)\right\| \rightarrow 0 .
$$

Indeed, since $\bar{B}(0, \varepsilon)$ is complete (notice that $\bar{B}_{h}(0, \varepsilon) \subset$ $\bar{B}(0, \varepsilon)),(12)$ leads to $z\left(\tau_{0}^{*}, \bar{u}, z_{0}\right) \in \bar{B}(0, \varepsilon)$. Then, by the uniqueness of the time optimal control, we deduce that $\bar{u}=u_{0}^{*}$.

Now we prove (12). We have :

$$
\begin{array}{r}
\left\|z_{h_{n}}\left(\tau_{h_{n}}^{*}, u_{h_{n}}^{*}, P_{h} z_{0}\right)-z\left(\tau_{0}^{*}, \bar{u}, z_{0}\right)\right\| \\
\leqslant\left\|z_{h_{n}}\left(\tau_{h_{n}}^{*}, u_{h_{n}}^{*}, P_{h} z_{0}\right)-z\left(\tau_{h_{n}}^{*}, u_{h_{n}}^{*}, z_{0}\right)\right\| \\
+\left\|z\left(\tau_{h_{n}}^{*}, u_{h_{n}}^{*}, z_{0}\right)-z\left(\tau_{0}^{*}, u_{h_{n}}^{*}, z_{0}\right)\right\| \\
+\left\|z\left(\tau_{0}^{*}, u_{h_{n}}^{*}, z_{0}\right)-z\left(\tau_{0}^{*}, \bar{u}, z_{0}\right)\right\| .
\end{array}
$$

Now we prove that these three parts converge to zero in order to deduce (12).

It is clear that (13) converges to zero using the error estimate (7).

Moreover, since $t \mapsto z\left(t, u, z_{0}\right)$ is continuous and $\tau_{h_{n}}^{*} \rightarrow \tau_{0}^{*},(14)$ converges to zero.

It remains to prove that (15) converges to zero. For that, denote $\psi(t)=z\left(t, \bar{u}, z_{0}\right)$ and $\psi_{n}(t)=z\left(t, u_{h_{n}}^{*}, z_{0}\right)$.

Then by Lemma 3 , we know that $\left(\psi_{n}\right)_{n}$ is a bounded sequence in :

$$
W=C\left(0, T ; X_{\frac{1}{2}-\alpha}\right) \cap L^{2}\left(0, T ; X_{1-\alpha}\right)
$$

$$
\cap W^{1,2}\left(0, T ; X_{-\alpha}\right) .
$$

Using a generalized Aubin-Lions Theorem (see [13, Cor. 4, p.85])) we deduce that :

$$
\begin{gathered}
\exists \widetilde{\psi} \in C([0, T] ; X) \text { s.t. } \\
\psi_{n} \rightarrow \tilde{\psi} \text { strongly in } C(0, T ; X)
\end{gathered}
$$

and

$$
\psi_{n} \rightarrow \tilde{\psi} \text { weakly in } W .
$$

Now we prove that $\widetilde{\psi}=\psi$. We know that $\left(\psi_{n}\right)_{n}$ satisfies :

$$
\begin{aligned}
\dot{\psi}_{n} & =A \psi_{n}+B u_{h_{n}}^{*}, \\
\psi_{n}(0) & =z_{0} .
\end{aligned}
$$

We prove then that $\dot{\psi}_{n} \rightarrow \dot{\widetilde{\psi}}$ weakly in $L^{2}\left(0, T ; X_{-\alpha}\right), A \psi_{n} \rightarrow A \widetilde{\psi}$ weakly in $L^{2}\left(0, T ; X_{-\alpha}\right)$, $B u_{h_{n}}^{*} \rightarrow B \bar{u}$ weakly in $L^{2}([0, T] ; X)$ and $\tilde{\psi}(0)=z_{0}$.

The first two convergences are clear since $\psi_{n} \rightarrow$ $\tilde{\psi}$ weakly in $W$.

Moreover, $u_{h_{n}}^{*} \stackrel{*}{\rightarrow} \bar{u}$ weakly $*$ in $L^{\infty}([0, T] ; U)$ implies that $u_{h_{n}}^{*} \rightarrow \bar{u}$ weakly in $L^{2}([0, T] ; U)$. Thus, $B u_{h_{n}}^{*} \rightarrow B \bar{u}$ in $L^{2}([0, T] ; X)$.

It remains to prove that $\tilde{\psi}(0)=z_{0}$. Indeed, we know $z_{0}=\psi_{n}(0) \rightarrow \tilde{\psi}(0)$, since $\psi_{n} \rightarrow$ $\tilde{\psi}$ strongly in $C(0, T ; X)$.

Consequently, $\tilde{\psi}$ satisfies :

$$
\begin{aligned}
\dot{\tilde{\psi}} & =A \tilde{\psi}+B \bar{u}, \\
\tilde{\psi}(0) & =z_{0},
\end{aligned}
$$

which implies that $\tilde{\psi}=\psi$.

This leads to the fact that (15) converges to zero.

Thus, we have :

$$
u_{h}^{*} \rightarrow u_{0}^{*} \text { weakly } * \text { in } L^{\infty}(0, T ; U) .
$$

We deduce immediately that :

$$
u_{h}^{*} \rightarrow u_{0}^{*} \text { weakly in } L^{2}(0, T ; U) .
$$

At last, since both $u_{h}^{*}$ and $u_{0}^{*}$ are bang-bang controls, we have $\lim _{h \rightarrow 0}\left\|u_{h}^{*}\right\|_{L^{2}(0, T ; U)}=\left\|u_{0}^{*}\right\|_{L^{2}(0, T ; U)}$. This leads to the strong convergence in $L^{2}(0, T ; U)$ and ends the proof.

\section{Example.}

We consider here 1 -D heat equation over $[0,1]$ with internal control over $\left[\frac{1}{3}, \frac{2}{3}\right]$, more precisely, for every $t \geq 0$,

$$
\begin{aligned}
\dot{z}(t, x) & =\partial_{x x}^{2} z(t, x) \\
& +\chi_{\left[\frac{1}{3}, \frac{2}{3}\right]}(x) u(t, x) \quad(x \in[0,1], t \geq 0), \\
z(t, 0) & =z(t, 1)=0, \quad(t \geq 0), \\
z(0, x) & =2 \sin (\pi x),(x \in[0,1]),
\end{aligned}
$$


where $\chi_{\left[\frac{1}{3}, \frac{2}{3}\right]}$ is the characteristic function of the interval $\left[\frac{1}{3}, \frac{2}{3}\right]$. Obviously, (17)-(19) has the form (1)-(2) by taking $A_{0}=-\partial_{x x}^{2}$ with Dirichlet boundary conditions of domain $D\left(A_{0}\right)=H_{0}^{1}(0,1) \cap H^{2}(0,1)$ on $X=L^{2}(0,1)$.

The control operator $B \in \mathscr{L}(U, X)$ (here $\alpha=0$ ) is defined by:

$$
B \varphi=\chi_{\left[\frac{1}{3}, \frac{2}{3}\right]} \tilde{\varphi},
$$

where $U=L^{2}\left(\frac{1}{3}, \frac{2}{3}\right)$ and where $\tilde{\varphi}$ is the extension of $\varphi$ outside $\left[\frac{1}{3}, \frac{2}{3}\right]$.

Now we consider the space semi-discrete approximation of (17)-(19) derived by the finite difference method. More precisely, for $N \in \mathbb{N}^{*}$ given and $h=\frac{1}{N+1}$, let $z_{i}(t)$ an approximation of $z(t, i h)$. We consider the following scheme:

$$
\begin{gathered}
\dot{z}_{i}(t)=\frac{z_{i+1}^{n}-2 z_{i}^{n}+z_{i-1}^{n}}{h^{2}}+B_{h} u_{i}(t), \\
z_{0}(t)=z_{N+1}(t)=0, \\
z_{i}(0)=2 \sin (\pi i h),
\end{gathered}
$$

where $B_{h} u_{i}=u_{i}$ if $i h \in\left[\frac{1}{3}, \frac{2}{3}\right]$ and 0 otherwise. If we denote the unknown $z_{h}(t)=\left(z_{i}(t)\right)_{1 \leqslant i \leqslant N}^{T}$, the above scheme can be rewritten in the vector form as (3)-(4).

It is well known that that $(C 1)-(C 4)$ are satisfied with $\beta=0$ and $\theta=1$ (see for example in [14])

According to Theorem 1 , for every $z_{0} \in X,\left\|z_{0}\right\|_{X} \geqslant$ $\varepsilon$, we have $\lim _{h \rightarrow 0} \tau_{h}^{*}=\tau_{0}^{*}$. We test this scheme in Matlab and have the following result:

\begin{tabular}{|c|c|c|c|c|c|c|}
\hline $\mathrm{N}$ & 5 & 10 & 20 & 30 & 40 & 50 \\
\hline$\tau_{h}^{*}$ & 0.0344 & 0.0336 & 0.0331 & 0.0332 & 0.0331 & 0.0330 \\
\hline
\end{tabular}

\section{References}

[1] N. ARADA AND J.-P. RAYMOND, Time optimal problems with Dirichlet boundary controls, Discrete Contin. Dyn. Syst., 9 (2003), pp. 1549-1570.

[2] M. BADRA,Stabilisation par feedback et approximation des équations de Navier-Stokes, Phd thesis supervised by Raymond, Jean-Pierre, (2006), ch5, http://www.theses.fr/2006TOU30242.

[3] V. BARBU, Analysis and control of nonlinear infinitedimensional systems, vol. 190 of Mathematics in Science and Engineering, Academic Press, Inc., Boston, MA, 1993.

[4] H.O. FAtToRini, Infinite Dimensional Optimization and Control Theory, Cambridge Studies in Advanced Mathematics, Cambridge University Press, 1999.

[5] H. O. FATTORINI, Time-optimal control of solutions of operational differenital equations, Journal of the Society for Industrial and Applied Mathematics Series A Control, 2 (1964), pp. 54-59.
[6] _ Infinite Dimensional Linear Control Systems. The Time Optimal and Norm Optimal Control Problems, North-Holland Mathematics Studies, 201, Elsevier, Amsterdam, 2005.

[7] G.P. Henze, C. Felsmann and G. Knabe, Evaluation of optimal control for active and passive building thermal storage, International Journal of Thermal Sciences, $\mathbf{4 3}$ (2004), pp. 173-183.

[8] K. KUNISCH AND L. WANG, Time optimal control of the heat equation with pointwise control constraints, ESAIM Control Optim. Calc. Var., 19 (2013), pp. 460485.

[9] I. Lasiecka And R. TRIggiani, Control Theory for Partial Differential Equations: Volume 1, Abstract Parabolic Systems: Continuous and Approximation Theories, Cambridge University Press (2000), ch4.

[10] X.J. LI AND J.M. Yong, Optimal control theory for infinite-dimensional systems, Systems \& Control: Foundations \& Applications, Birkhäuser Boston Inc., Boston, MA, 1995.

[11] F. TRÖLTZSCH, On generalized bang-bang principles for two time-optimal heating problems with constraints on the control and the state, Demonstratio Math., 15 (1982), pp. 131-143.

[12] M. TUCSNAK AND G. WeISS, Observation and control for operator semigroups, Birkhäuser Advanced Texts: Basler Lehrbücher. [Birkhäuser Advanced Texts: Basel Textbooks], Birkhäuser Verlag, Basel, 2009.

[13] J. Simon,Compact sets in the space $L^{p}(0, T ; B)$, Annali di Matematica Pura ed Applicata. Serie Quarta, (1987), pp. 65-96.

[14] T. VIDAR, Galerkin finite element methods for parabolic problems, Springer Series in Computational Mathematics, Berlin, 50 (1997).

[15] G. WANG AND L. WANG, Finite element approximations of optimal controls for the heat equation with endpoint state constraints, Int. J. Numer. Anal. Model., 9 (2012), pp. 844-875.

[16] G. WANG AND G. ZHENG, An approach to the optimal time for a time optimal control problem of an internally controlled heat equation, SIAM J. Control Optim., 50 (2012), pp. 601-628. 